IMA Journal of Numerical Analysis (2016) 36, 1167-1192

doi:10.1093/imanum/drv043

Advance Access publication on September 14, 2015

\title{
Nonsmooth trust region algorithms for locally Lipschitz functions on Riemannian manifolds
}

\author{
P. GROHS AND S. HosseINI* \\ ETH Zürich, Seminar for Applied Mathematics, Rämistrasse 101, 8092 Zürich, Switzerland \\ *Corresponding author: seyedehsomayeh.hosseini@math.ethz.ch pgrohs@sam.math.ethz.ch
}

[Received on 13 October 2014; revised on 1 April 2015]

\begin{abstract}
This paper presents a Riemannian trust region algorithm for unconstrained optimization problems with locally Lipschitz objective functions defined on complete Riemannian manifolds. To this end we define a function $\Phi: T M \rightarrow \mathbb{R}$ on the tangent bundle $T M$, and at the $k$ th iteration, using the restricted function $\left.\Phi\right|_{T_{x_{k}} M}$, where $T_{x_{k}} M$ is the tangent space at $x_{k}$, a local model function $Q_{k}$ that carries both first- and second-order information for the locally Lipschitz objective function $f: M \rightarrow \mathbb{R}$ on a Riemannian manifold $M$, is defined and minimized over a trust region. We establish the global convergence of the proposed algorithm. Moreover, using the Riemannian $\varepsilon$-subdifferential, a suitable model function is defined. Numerical experiments illustrate our results.
\end{abstract}

Keywords: Riemannian manifolds; Lipschitz function; trust region method; Clarke subdifferential.

\section{Introduction}

Most classical problems considered in optimization are formulated in Banach spaces, where the linear structure plays an important role. However, many problems in computer vision, robotics, signal processing and geometric mechanics, to name but a few, are more conveniently expressed as optimization problems on Riemannian manifolds (Riddell, 1984; Mahony, 1996; Adler et al., 2002; Lee, 2005; Sarkis \& Diepold, 2012; Usevich \& Markovsky, 2014). Therefore, it is of eminent interest to develop useful computational and theoretical tools of optimization on manifolds. This paper is concerned with the numerical solution of optimization problems defined on Riemannian manifolds, where the objective function may be nonsmooth.

Many algorithms for solving the following unconstrained optimization problem,

$$
\min _{x \in \mathbb{R}^{n}} f(x),
$$

where $f: \mathbb{R}^{n} \rightarrow \mathbb{R}$ is continuously differentiable, have been proposed. Trust region methods are an important class of iterative methods due to their strong global convergence and fast local convergence; see Conn et al. (2000). In this class of iterative methods, a step to the $k+1$ th iterate is obtained by minimizing a model function $Q_{k}$ defined by

$$
Q_{k}\left(x_{k}, d\right)=f\left(x_{k}\right)+\nabla f\left(x_{k}\right)^{T} d+\frac{1}{2} d^{T} B_{k} d,
$$

over a restricted region centred at the current iterate. It is worth pointing out that in this model function, $B_{k}$ is adequately selected, and the model function preserves the first- and second-order information of the objective function $f$. The so-called trust region ratio evaluates an agreement between the model and the actual objective reductions along the computed step. Considering the trust region ratio, one can 
decide whether the step is accepted or rejected. After that the trust region radius is updated and a new point is obtained.

The classical trust region methods for smooth problems cannot be used for nonsmooth ones since, in general, the gradient of the objective function at the current iterate does not exist. Several trust region methods for minimizing a nonsmooth objective function defined on a linear space have been presented and applied to the nonlinear equations problem, the nonlinear fitting problem and the constrained optimization problems; see Qi \& Sun (1994), Dennis et al. (1995), Akbari et al. (2015) and references therein. The most well-known nonsmooth trust region methods begin from a starting point $x_{1}$, which may not be close to the minimum of the objective function $f: \mathbb{R}^{n} \rightarrow \mathbb{R}$. They use a function $\Phi: \mathbb{R}^{n} \times \mathbb{R}^{n} \rightarrow \mathbb{R}$ to build at each iteration a model $Q_{k}$ defined by

$$
Q_{k}\left(x_{k}, d\right)=f\left(x_{k}\right)+\Phi\left(x_{k}, d\right)+\frac{1}{2} d^{T} B_{k} d,
$$

which must be an approximation of $f\left(x_{k}+d\right)$ for small $d$. Therefore, they should impose some conditions on the function $\Phi: \mathbb{R}^{n} \times \mathbb{R}^{n} \rightarrow \mathbb{R}$ and the sequence of the symmetric matrices $B_{k}$. For instance, Qi \& Sun (1994) is among the first works on trust region methods for unconstrained optimization problems with locally Lipschitz objective functions; in that article several conditions on $B_{k}$ and $\Phi: \mathbb{R}^{n} \times \mathbb{R}^{n} \rightarrow \mathbb{R}$ are proposed, which ensure that $Q_{k}$ is an approximation for $f\left(x_{k}+d\right)$ for small $d$ and the algorithm is convergent.

Nonsmooth trust region algorithms approximately solve the subproblem

$$
\min _{\left\{d \in \mathbb{R}^{n}:\|d\| \leqslant \delta_{k}\right\}} Q_{k}\left(x_{k}, d\right)
$$

to obtain $d_{k}$. Using the trust region ratio, either the step is accepted or rejected.

The extension of nonsmooth trust region algorithms and their (global) convergence properties to Riemannian manifolds are the subject of the present paper. A manifold, in general, does not have a linear structure; hence the usual techniques, which are often used to study optimization problems on linear spaces, cannot be applied and new techniques need to be developed.

There is clearly a link between the techniques of optimization on manifolds and standard constrained optimization approaches. However, there are manifolds that are not defined as constrained sets in $\mathbb{R}^{n}$; important examples are Grassmann manifolds (Ashikhmin \& Calderbank, 2010; Ozbek \& Ruyet, 2013) or symmetric positive definite matrices (Basser et al., 1994; Pennec et al., 2006; Weinmann et al., 2014). To solve optimization problems on these spaces, intrinsic methods are a popular method of choice.

A manifold is, per definition, locally isomorphic to a linear space via chart maps. For this reason, one might wonder whether it suffices to simply work in a chart domain and use classical linear algorithms. Unfortunately, such an approach does in general not lead to useful algorithms: first of all, symmetries of the underlying Riemannian manifold will in general not be respected by such algorithms. Moreover, there often does not exist a canonical useful representation for charts. But more fundamentally, localizing to a chart inevitably leads to distortions in the metric which leads to much slower convergence. Finally, we are interested in establishing global convergence of the algorithms which we study. Such a property is clearly out of reach by working in a chart domain, which is a local procedure (it should now also be clear that the mathematical analysis of global convergence requires global arguments from Riemannian geometry and cannot be deduced from corresponding linear results). For all those reasons, together with the evident need for efficient and reliable Riemannian optimization algorithms, the construction and study of intrinsic algorithms has become a thriving area of research in the past few years. 
The development of Riemannian nonsmooth optimization algorithms is primarily motivated by large-scale applications that have gained much popularity in recent years regarding the framework of $l^{1}$ optimization, stochastic optimization and statistical learning. Those applications include robust, sparse, structured principal component analysis, statistics on manifolds (e.g., median calculation of positive semidefnite tensors) and low-rank optimization (matrix completion, collaborative filtering, source separation); see Kressner et al. (2014), Vandereycken \& Vandewalle (2010), Vandereycken (2013) and Usevich \& Markovsky (2014). Furthermore, these algorithms have many applications in image processing, computer vision, nonsmooth constrained optimization problems on linear spaces; Afsari et al. (2013), Borckmans et al. (2014) and Dirr et al. (2007).

Previous work. For the optimization of smooth objective functions, many classical methods for unconstrained minimization, such as Newton-type and trust-region methods, have been successfully generalized to problems on Riemannian manifolds; see Absil \& Baker (2007), Adler et al. (2002), da Cruz Neto et al. (1998), Mahony (1996), Ring \& Wirth (2012), Smith (1994), Udriste (1994) and Zhang (2010). The recent monograph by Absil et al. (2008) discusses, in a systematic way, the framework and many numerical first- and second-order manifold-based algorithms for minimization problems on Riemannian manifolds with an emphasis on applications to numerical linear algebra; see Absil et al. (2008).

As we discussed above, one of the most important methods in the unconstrained optimization of smooth functions is the trust region method, due to its strong global convergence and fast local convergence. In Absil \& Baker (2007), a Riemannian trust region method for smooth functions on Riemannian manifolds was introduced. Similar to Euclidean trust region methods, the Riemannian trust region method ensures global convergence properties while allowing superlinear local convergence. The trick in Absil \& Baker (2007) was to define a retraction $R$ on a Riemannian manifold $M$ that defines, for any $x \in M$, a one-to-one correspondence $R_{x}$ between a neighbourhood of $x$ in $M$ and a neighbourhood of $0_{x}$ in the tangent space $T_{x} M$. Using this retraction, the objective function $f$ on $M$ is lifted to an objective function $\overline{f_{x}}=f \circ R_{x}$ on $T_{x} M$. Then, a quadratic model of $\overline{f_{x}}$ is defined, and a classical method on the Euclidean space $T_{x} M$ is used to compute a minimizer of the model within a trust region around $0_{x} \in T_{x} M$. Afterwards, the minimizer is lifted back to $M$ to be a new candidate for the next iterate. The most noticeable point in the mentioned trust region method is that it does not deal with a unique objective function, since in any iteration the retraction is changed. However, the authors proved that under some conditions, the nice properties of the classical trust region method are preserved in their Riemannian generalizations.

In considering optimization problems with nonsmooth objective functions on Riemannian manifolds, it is necessary to generalize concepts of nonsmooth analysis to Riemannian manifolds. In the past few years, a number of results have been obtained on numerous aspects of nonsmooth analysis on Riemannian manifolds; see Azagra et al. (2005), Azagra \& Ferrera (2007), Hosseini \& Pouryayevali (2011, 2013a,b) and Li et al. (2011).

Recently, some mathematicians have started developing nonsmooth optimization algorithms for manifold settings, although their attempts are limited to generalizing some subgradient-based and proximal point algorithms. In Bento et al. $(2010,2015)$ and Ferreira \& Oliveira (2002), constrained minimization problems on Hadamard manifolds are solved using a generalization of the proximal point method. There have also been some studies by Ferreira, Bento and Oliveira, and their colleagues who generalized subgradient-type methods for convex and quasiconvex functions defined on Riemannian manifolds; see Bento et al. (2012), Bento \& Melo (2012), da Cruz Neto et al. (1999), Ferreira \& Oliveira (1998) and Papa Quiroz et al. (2008). Finally, it is worth mentioning the work of Dirr et al. (2007), which presents a survey on Riemannian geometry methods for smooth and nonsmooth constrained 
optimization, as well as gradient and subgradient descent algorithms on a Riemannian manifold. In that paper, the methods are illustrated by applications from robotics and multi-antenna communication.

Contributions. Our main contributions are twofold. First, we impose some conditions on a function $\Phi: T M \rightarrow \mathbb{R}$, where $T M$ is the tangent bundle of a Riemannian manifold $M$, to ensure that our model function

$$
Q_{k}\left(x_{k}, \cdot\right)=f\left(x_{k}\right)+\Phi\left(x_{k}, \cdot\right)+\frac{1}{2}\left\langle B_{k} \cdot, \cdot\right\rangle: T_{x_{k}} M \rightarrow \mathbb{R}
$$

preserves the first-order information of the locally Lipschitz objective function $f: M \rightarrow \mathbb{R}$. Our proposed conditions are generalizations of the conditions in Qi \& Sun (1994). Our first main result, Theorem 3.9, states that, provided these assumptions are satisfied, then the Riemannian trust region scheme which iteratively (approximately) minimizes the model function $Q_{k}$ on a ball in $T_{x_{k}} M$ converges globally to a critical point of $f$.

As the second main contribution of this paper, in Section 4 we propose several choices for a suitable function $\Phi$, the simplest one being the Clarke generalized directional derivative of $f$ as a function $T M \rightarrow$ $\mathbb{R}$. However, this function is only practical if the subdifferential of $f$ can be computed explicitly, which might not be the case. Therefore, based on an approximation scheme for the so-called $\varepsilon$-subdifferential first introduced in Grohs \& Hosseini (2015) for the Riemannian case, we also present a numerical approximation of the subdifferential which is efficiently computable and which still yields a globally convergent algorithm. This is carried out in Section 4.

To the best of our knowledge, the resulting algorithm is the first practical nonsmooth trust region algorithm for locally Lipschitz functions defined on Riemannian manifolds. In Section 5 we present some numerical experiments. Our proposed algorithm is implemented in MATLAB and applied to some nonsmooth problems with locally Lipschitz objective functions. Numerical results show that the proposed algorithm has a far better performance in some problems in comparison with existing first-order methods, such as subgradient descent.

\section{Preliminaries}

Throughout this paper, $M$ is an $n$-dimensional complete manifold endowed with a Riemannian metric $\langle\cdot, \cdot\rangle$ on the tangent space $T_{x} M$; see Lang (1999). As usual, $B(x, \delta)$ denotes the open ball with respect to the Riemannian distance centred at $x$ with radius $\delta$. For the point $x \in M$, $\exp _{x}: U_{x} \rightarrow M$ defines the exponential function at $x$, where $U_{x}$ is an open subset of $T_{x} M$. For a minimizing geodesic $\gamma:[0, l] \rightarrow M$ connecting $x$ to $y$ in $M$, and for a vector $v \in T_{x} M$ there is a unique parallel vector field $P$ along $\gamma$ such that $P(0)=v$; this is called the parallel translation of $v$ along $\gamma$. The mapping $T_{x} M \ni v \mapsto P(l) \in T_{y} M$ is a linear isometry from $T_{x} M$ onto $T_{y} M$. This map is denoted by $L_{x y}$. An easy consequence of the definition of the parallel translation along a curve as a solution to an ordinary linear differential equation implies that the mapping

$$
C: T M \rightarrow T_{x_{0}} M, \quad C(x, \xi)=L_{x x_{0}}(\xi),
$$

when $x$ is in a neighbourhood $U$ of $x_{0}$, is well-defined and continuous at $\left(x_{0}, \xi_{0}\right)$; that is, if $\left(x_{n}, \xi_{n}\right) \rightarrow$ $\left(x_{0}, \xi_{0}\right)$ in $T M$, then $L_{x_{n} x_{0}}\left(\xi_{n}\right) \rightarrow L_{x_{0} x_{0}}\left(\xi_{0}\right)=\xi_{0}$, for every $\left(x_{0}, \xi_{0}\right) \in T M$; see Azagra et al. (2005, Remark 6.11).

Note that $i_{M}(x)$ denotes the injectivity radius of $M$ at $x$; that is, the supremum of the radius $r$ of all balls $B\left(0_{x}, r\right)$ in $T_{x} M$ for which $\exp _{x}$ is a diffeomorphism from $B\left(0_{x}, r\right)$ onto $B(x, r)$. If $U$ is a compact subset of a Riemannian manifold $M$ and $i(U):=\inf \left\{i_{M}(x): x \in U\right\}$, then $0<i(U)$; see Klingenberg (1995). 
A set $S$ in a Riemannian manifold $M$ is said to be convex if every two points $p_{1}, p_{2} \in S$ can be joined by a unique geodesic whose image belongs to $S$.

A real-valued function $f$ is said to be locally Lipschitz on $M$ if $f$ is Lipschitz near $x$ for every $x \in M$; that is, for every $x \in M$, there exist an open neighbourhood $B(x, \delta)$ and a real number $k>0$ such that $|f(z)-f(y)| \leqslant k \operatorname{dist}(z, y)$ for every $z, y \in B(x, \delta)$, where dist is the Riemannian distance on $M$.

It is easy to see that the metric on TM can be defined as follows; see Canary et al. (2006). Assume that $u, v \in T M, \pi: T M \rightarrow M$ is the projection map, and let $\gamma$ be a piecewise smooth path from $x=\pi(u)$ to $y=\pi(v)$, whose derivative is never zero. Let $L_{x y}^{\gamma}$ be the parallel translation along $\gamma$. We define the square of the distance from $u$ to $v$ as the infimum over all paths $\gamma$ from $\pi(u)$ to $\pi(v)$ of

$$
\operatorname{dist}_{\gamma}(u, v)^{2}=\left\|L_{x y}^{\gamma}(u)-v\right\|^{2}+\operatorname{length}(\gamma)^{2} .
$$

To justify the above assertion, note that, over a small piece of $\gamma$, parallel translation gives us a canonical identification of any two tangent spaces, and hence the tangent bundle is metrically the product of an interval and $\mathbb{R}^{n}$.

If $x$ and $y$ are two points in the same convex neighbourhood in $M$, let $\gamma$ be the geodesic joining them and $L_{x y}$ be the parallel translation along $\gamma$ from the tangent space at $x$ to the tangent space at $y$. Then,

$$
\operatorname{dist}_{T M}(u, v)^{2} \leqslant\left\|L_{x y}(u)-v\right\|^{2}+\operatorname{dist}(x, y)^{2} .
$$

Moreover,

$$
\operatorname{dist}(x, y) \leqslant \operatorname{dist}_{T M}(u, v) .
$$

\section{A nonsmooth trust region method on Riemannian manifolds}

Assume that $f: M \rightarrow \mathbb{R}$ is a locally Lipschitz function on a complete Riemannian manifold $M$.

The trust region algorithm proposed in the present section, which is based on Qi \& Sun (1994), relies on the choice of a function $\Phi: T M \rightarrow \mathbb{R}$ (modelling the derivative of $f$ ) and a sequence $\left\{B_{k}: k=1,2, \ldots\right\}$ of $n \times n$ symmetric matrices (modelling the Hessian of $f$ ) from which we build a sequence of model functions

$$
Q_{k}: \begin{cases}T_{x_{k}} M & \rightarrow \mathbb{R} \\ \left(x_{k}, d\right) & \mapsto f\left(x_{k}\right)+\Phi\left(x_{k}, d\right)+\frac{1}{2}\left\langle B_{k} d, d\right\rangle,\end{cases}
$$

analogous to a second-order Taylor expansion in the Euclidean case.

Then, the proposed trust region scheme iteratively computes approximative minima of these model functions over a trust region as follows:

Let

$$
d_{k}^{*}=\operatorname{argmin}\left\{Q_{k}\left(x_{k}, d_{k}\right)=f\left(x_{k}\right)+\Phi\left(x_{k}, d_{k}\right)+1 / 2\left\langle B_{k} d_{k}, d_{k}\right\rangle: d_{k} \in T_{x_{k}} M,\left\|d_{k}\right\| \leqslant \delta_{k}\right\} .
$$

Then in each iteration step we compute $d_{k}^{*}$ or, if this turns out to be not practical, an approximation $\bar{d}_{k}$ of $d_{k}^{*}$ in the sense that

$$
f\left(x_{k}\right)-Q_{k}\left(x_{k}, \bar{d}_{k}\right) \geqslant c_{0}\left[f\left(x_{k}\right)-Q_{k}\left(x_{k}, d_{k}^{*}\right)\right] \text { and }\left\|\bar{d}_{k}\right\| \leqslant \delta_{k}
$$

for a fixed constant $0<c_{0} \leqslant 1$. 
Then, depending on the trust ratio in each step, we either reduce the trust region by reducing the value $\delta_{k}$ and solving (3.1), or we update

$$
x_{k+1}:=\exp _{x_{k}}\left(\bar{d}_{k}\right)
$$

see Algorithm 1.

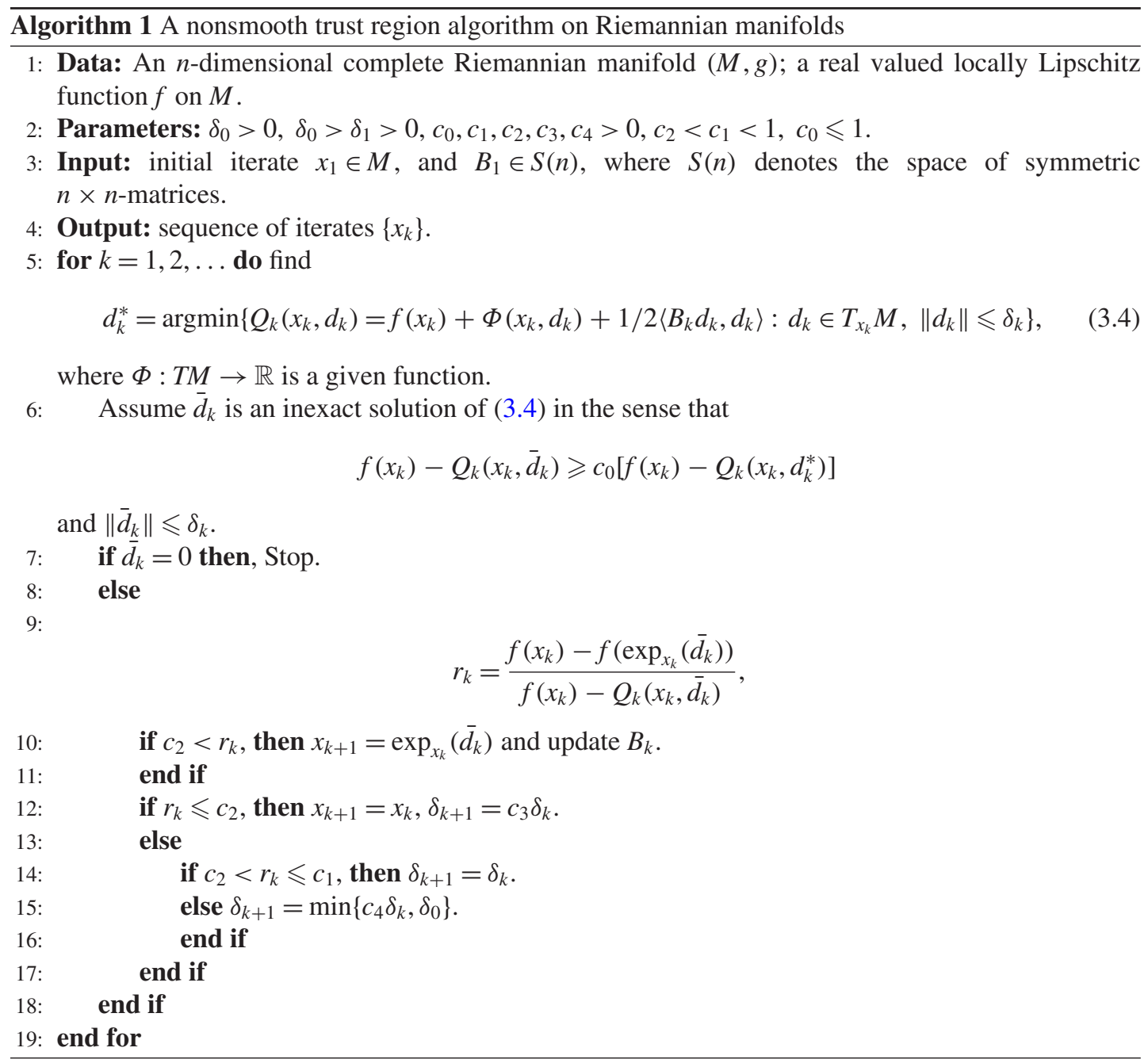

REMARK 3.1 A natural choice for the function $\Phi$ would be the Clarke generalized directional derivative of $f$ as defined below in (4.1).

In case the Clarke generalized directional derivative of $f$ is not given explicitly (as is the case, for instance, in Dirr et al., 2007, Sections 3.2 and 3.3; Lageman \& Helmke, 2007), in Section 4 we also propose a numerical approximation which only solves (3.1). The choice of matrices $\left\{B_{k}\right\}$ is quite general; 
in our results they simply need to be uniformly bounded, but even this property can be weakened; see, for instance, Qi \& Sun (1994). In our algorithms we have used a Riemannian generalization of the BFGS method to iteratively update the matrices $B_{k}$; see also Qi et al. (2010).

REMARK 3.2 Instead of using the exponential map to update $x_{k}$, we can choose a retraction $R: T M \rightarrow M$. The notion of retraction on a manifold includes all first-order approximations to the Riemannian exponential; see Absil \& Baker (2007). The retraction can be used to take a step in the direction of a tangent vector. Using a good retraction amounts to finding an approximation of the exponential mapping that can be computed with low computational cost while not adversely affecting the behaviour of the optimization algorithm.

In the remainder of this section we formulate assumptions on the $\Phi$ to ensure global convergence of the sequence $\left(x_{k}\right)_{k}$ to a critical point of $f$, in the following sense.

Definition 3.3 With $f: M \rightarrow \mathbb{R}$ and $\Phi: T M \rightarrow \mathbb{R}$, define

$$
\psi(x, \delta)=\sup \left\{-\Phi(x, d): d \in T_{x} M,\|d\| \leqslant \delta\right\} .
$$

The point $x \in M$ is called a critical point with respect to $\Phi$ of the objective function $f$ if there exists $\delta>0$ such that $\psi(x, \delta)=0$.

In the Euclidean case, the notion of critical point as defined in Definition 3.3 is common in the context of nonsmooth trust region methods; see Qi \& Sun (1994) and Dennis et al. (1995). Note that the upper-Dini directional derivative of $f$ at $x$ in the direction $d \in T_{x} M$ denoted by $f^{+}(x ; d)$ is defined as follows:

$$
f^{+}(x ; d):=\limsup _{t \downarrow 0} \frac{f\left(\exp _{x}(t d)\right)-f(x)}{t} .
$$

A point $x$ is called a Dini stationary point if, for all $d \in T_{x} M, f^{+}(x ; d) \geqslant 0$. Under weak assumptions, a critical point corresponds to a so-called 'Dini stationary point' (Qi \& Sun, 1994). Indeed, we may impose the following assumption on $\Phi$ in order to be able to prove that any critical point of $f$ is also a Dini stationary point of $f$.

Assumption 3.4 Assume that $D$ is a bounded open convex set containing $N:=\left\{x \in M: f(x) \leqslant f\left(x_{0}\right)\right\}$ and, for all $x \in D$ and $d \in T_{x} M$, it holds that

$$
\liminf _{t \downarrow 0} \frac{\Phi(x, t d)}{t} \leqslant f^{+}(x ; d),
$$

where $f^{+}(x ; d)$ is the upper-Dini directional derivative of $f$ at $x$ in the directional $d \in T_{x} M$.

It is obvious that if $x$ is a critical point of $f$ in the sense of Definition 3.3, then, for $t$ small enough, $\Phi(x, t d) \geqslant 0$ and therefore $\liminf _{t \downarrow 0}(\Phi(x, t d) / t) \geqslant 0$. Hence, using Assumption 3.4, we have that, for all $d \in T_{x} M, f^{+}(x ; d) \geqslant 0$. One can also show that a local minimizer $x$ of a locally Lipschitz function $f: M \rightarrow \mathbb{R}$ is always a critical point, provided that the function $\Phi$ satisfies some natural assumption. This will be done in Lemma 3.6.

\subsection{Convergence conditions}

Let $\Phi: T M \rightarrow \mathbb{R}$ and $I=\{1,2, \ldots\}, N=\left\{x \in M: f(x) \leqslant f\left(x_{1}\right)\right\}$, where $x_{1}$ is a starting point for Algorithm 1. Let $\left\{B_{k}: k=1,2, \ldots\right\}$ be a sequence of $n \times n$ symmetric matrices. In this section, we 
make some assumptions on $\Phi$ and $B_{k}$, and show that these assumptions ensure global convergence of Algorithm 1 to a critical point of $f$ in the sense of Definition 3.3. We extend results of Qi \& Sun (1994) to the Riemannian case.

We start with the following assumptions on the function $\Phi$.

Assumption 3.5 Let $\Phi: T M \rightarrow \mathbb{R}$. Assume that

$$
\begin{gathered}
\qquad\left(x, 0_{x}\right)=0 \quad \forall x \in M, \\
\Phi(x, \alpha d) \leqslant \alpha \Phi(x, d) \quad \forall(x, d) \in T M, \quad 0 \leqslant \alpha \leqslant 1, \\
\text { for all } x \in M,\left.\Phi\right|_{T_{x} M} \text { is lower-semicontinuous; }
\end{gathered}
$$

for any $(x, d) \in T M$ it holds that

$$
f\left(\exp _{x}(d)\right)-f(x) \leqslant \Phi(d)+o(\|d\|)
$$

and there exists $\delta_{*}$ such that

$$
\text { for all } \delta<\delta_{*} \text { the function } \psi(\cdot, \delta) \text { is lower-semicontinuous, }
$$

where $\psi$ is defined in (3.5) and the implicit constant in the $o$-term is uniform over compact sets.

First, we show that, provided $\Phi$ satisfies Assumption 3.5, every local minimum of a locally Lipschitz function $f: M \rightarrow \mathbb{R}$ is a critical point in the sense of Definition 3.3.

Lemma 3.6 Suppose that $f: M \rightarrow \mathbb{R}$ and $\Phi: T M \rightarrow \mathbb{R}$ such that Assumption 3.5 holds. Then, every local minimizer of $f$ is a critical point in the sense of Definition 3.3.

In order to establish this result, we utilize the following simple lemma, which is a straightforward extension of (Qi \& Sun, 1994, Lemma 3.1).

Lemma 3.7 Suppose that $\Phi$ satisfies Assumption 3.5 and let $\psi$ be defined by (3.5). Then, for any $x \in M$, the function $d \mapsto \psi(x, d), d \in T_{x} M$, is nonnegative and nondecreasing and, for any $\alpha \in[0,1]$,

$$
\psi(x, \alpha \delta) \geqslant \alpha \psi(x, \delta),
$$

and for any $\delta>0$,

$$
\psi(x, \delta)=0 \quad \text { if and only if } \psi(x, 1)=0 .
$$

We can now proceed to the proof.

Proof of Lemma 3.6. Suppose that $\bar{x}$ is a local minimizer of $f$ on some neighbourhood $B(\bar{x}, \delta)$ which is not a critical point; then Lemma 3.7 implies that $\psi(\bar{x}, 1)>0$. Therefore, there exists $d_{\bar{x}}$ with $\left\|d_{\bar{x}}\right\| \leqslant 1$ such that $\Phi\left(\bar{x}, d_{\bar{x}}\right)<0$. Assume that $t<1$ is small enough; hence, by Assumptions (3.9) and (3.7),

$$
f\left(\exp _{\bar{x}}\left(t d_{\bar{x}}\right)\right)-f(\bar{x}) \leqslant \Phi\left(\bar{x}, t d_{\bar{x}}\right)+o\left(\left\|t d_{\bar{x}}\right\|\right) \leqslant t \Phi\left(\bar{x}, d_{\bar{x}}\right)+o\left(\left\|t d_{\bar{x}}\right\|\right) .
$$

Therefore, if $t$ is small enough,

$$
\frac{f\left(\exp _{\bar{x}}\left(t d_{\bar{x}}\right)\right)-f(\bar{x})}{t} \leqslant \Phi\left(\bar{x}, d_{\bar{x}}\right) / 2<0,
$$

which means that $\bar{x}$ is not a local minimizer of $f$ and this is a contradiction. 
Now, we want to establish conditions for the convergence of Algorithm 1 to a critical point of $f$.

We need to make more assumptions on the starting point and the sequence $\left(B_{k}\right)_{k}$.

Assumption 3.8 Recall $N=\left\{x \in M: f(x) \leqslant f\left(x_{1}\right)\right\}$, where $x_{1}$ is the starting point of Algorithm 1 . Assume that $N$ is bounded. Furthermore, assume that there exists $C>0$ such that $\left\|B_{k}\right\| \leqslant C$ for all $k=1,2, \ldots$

The main theorem of this section reads as follows.

Theorem 3.9 Suppose that $\Phi$ and $\left(B_{k}\right)_{k}$ are such that Assumptions 3.5 and 3.8 hold true. If $\bar{x}$ is an accumulation point of $\left\{x_{k}\right\}$, generated by Algorithm 1, then $\bar{x}$ is a critical point of $f$ in the sense of Definition 3.3.

In order to establish Theorem 3.9, we require the following result, which can be proved exactly in the same way as Lemma 3.2 in Qi \& Sun (1994).

Lemma 3.10 Let $x \in M$ and $\delta>0,0<c_{0} \leqslant 1$. Define

$$
d^{*}:=\operatorname{argmin}\left\{Q_{x}(d):=f(x)+\Phi(x, d)+1 / 2\langle B d, d\rangle: d \in T_{x} M,\|d\| \leqslant \delta\right\}
$$

and let $\bar{d}$ be an approximate solution of the above problem in the sense that

$$
f(x)-Q_{x}(\bar{d}) \geqslant c_{0}\left[f(x)-Q_{x}\left(d^{*}\right)\right] \quad \text { and } \quad\|\bar{d}\| \leqslant \delta .
$$

Then, for all $v \geqslant \delta$ it holds that

$$
f(x)-Q_{x}(\bar{d}) \geqslant \frac{c_{0}}{2 v} \psi(x, v) \min \{\delta, \psi(x, v) /(\|B\| v)\}
$$

where the second term in the min notation is understood as $\infty$ if $B=0$.

The following lemma proves that if Algorithm 1 generates a sequence $\left\{x_{k}\right\}$ with $x_{k}=\bar{x}$ for all large $k$, then $\bar{x}$ is a critical point of $f$.

Lemma 3.11 Suppose that $\bar{x}$ is an accumulation point of $\left\{x_{k}\right\}$ which is not a critical point. Then, there exist $\epsilon>0$ and $\beta>0$ such that, for all $k$ satisfying

$$
\operatorname{dist}\left(x_{k}, \bar{x}\right)<\epsilon, \quad 0<\delta_{k}<\beta, \quad\left\|B_{k}\right\| \leqslant C,
$$

we have

$$
r_{k}=\frac{f\left(x_{k}\right)-f\left(\exp _{x_{k}}\left(\bar{d}_{k}\right)\right)}{f\left(x_{k}\right)-Q_{k}\left(x_{k}, \bar{d}_{k}\right)}>c_{2},
$$

where $x_{k}, \delta_{k}, c_{2}$ are the same as in Algorithm 1.

Proof. Since $\bar{x}$ is not a critical point, Lemma 3.7 implies that $\psi(\bar{x}, \bar{\delta})>0$ for every $\bar{\delta}>0$. Using Assumption (3.10), let $\delta_{*}$ be such that, for all $0 \leqslant \delta \leqslant \delta_{*}, \psi(\cdot, \delta)$ is lower-semicontinuous. Therefore, 
there exist $\theta>0$ and $\epsilon>0$ such that $\psi\left(\bar{x}, \delta_{*}\right)=2 \theta>0$ and

$$
\psi\left(x_{k}, \delta_{*}\right)>\theta \text { provided } \operatorname{dist}\left(x_{k}, \bar{x}\right)<\epsilon .
$$

Assuming that $\delta_{k}<\beta<\delta_{*}$, then, by Assumption (3.9) and Lemma 3.10,

$$
\begin{aligned}
\frac{f\left(\exp _{x_{k}}\left(\bar{d}_{k}\right)\right)-Q_{k}\left(x_{k}, \bar{d}_{k}\right)}{f\left(x_{k}\right)-Q_{k}\left(x_{k}, \bar{d}_{k}\right)} & \leqslant \frac{o\left(\left\|\bar{d}_{k}\right\|\right)-1 / 2\left\langle B_{k} \bar{d}_{k}, \bar{d}_{k}\right\rangle}{\left(c_{0} / 2 \delta_{*}\right) \psi\left(x_{k}, \delta_{*}\right) \min \left\{\delta_{k}, \psi\left(x_{k}, \delta_{*}\right) /\left(\left\|B_{k}\right\| \delta_{*}\right)\right\}} \\
& \leqslant \frac{o\left(\left\|\bar{d}_{k}\right\|\right)+1 / 2\left\|B_{k}\right\|\left\|\bar{d}_{k}\right\|^{2}}{\left(c_{0} / 2 \delta_{*}\right) \psi\left(x_{k}, \delta_{*}\right) \min \left\{\delta_{k}, \psi\left(x_{k}, \delta_{*}\right) /\left(\left\|B_{k}\right\| \delta_{*}\right)\right\}} \\
& \leqslant \frac{o\left(\left\|\bar{d}_{k}\right\|\right)+1 / 2 C\left\|\bar{d}_{k}\right\| \delta_{*}}{\left(c_{0} / 2 \delta_{*}\right) \psi\left(x_{k}, \delta_{*}\right) \min \left\{\delta_{k}, \psi\left(x_{k}, \delta_{*}\right) /\left(\left\|B_{k}\right\| \delta_{*}\right)\right\}} \\
& \leqslant \frac{o\left(\delta_{k}\right)}{\left(c_{0} / 2 \delta_{*}\right) \theta \min \left\{\delta_{k}, \theta /\left(C \delta_{*}\right)\right\}} .
\end{aligned}
$$

Assuming that $\beta$ is small enough, then

$$
\frac{f\left(\exp _{x_{k}}\left(\bar{d}_{k}\right)\right)-Q_{k}\left(x_{k}, \bar{d}_{k}\right)}{f\left(x_{k}\right)-Q_{k}\left(x_{k}, \bar{d}_{k}\right)}<1-c_{2},
$$

which completes the proof.

We can now proceed to the proof.

Proof of Theorem 3.9. Note that $M$ is a complete Riemannian manifold and $N$ is compact. We assume that an infinite subsequence $\left\{x_{k}: k \in I_{*}\right\}$ converges to some $\bar{x}$ which is not a critical point. Let $I_{0}=\left\{k: r_{k}>c_{2}\right\}$ and $I_{*} \subset I_{0}$. Therefore, by Lemma 3.10

$$
\begin{aligned}
f\left(x_{k}\right)-f\left(x_{k+1}\right) & \geqslant c_{2}\left(f\left(x_{k}\right)-Q_{k}\left(x_{k}, d_{k}\right)\right) \\
& \geqslant \frac{c_{0} c_{2}}{2 \delta_{0}} \psi\left(x_{k}, \delta_{0}\right) \min \left\{\delta_{k}, \psi\left(x_{k}, \delta_{0}\right) /\left(\left\|B_{k}\right\| \delta_{0}\right)\right\} \\
& \geqslant \frac{c_{0} c_{2}}{2 \delta_{0}} \psi\left(x_{k}, \delta_{0}\right) \min \left\{\delta_{k}, \psi\left(x_{k}, \delta_{0}\right) /\left(C \delta_{0}\right)\right\} .
\end{aligned}
$$

Therefore,

$$
+\infty>f\left(x_{0}\right)-f(\bar{x}) \geqslant \frac{c_{0} c_{2}}{2 \delta_{0}} \sum_{k \in I_{0}} \psi\left(x_{k}, \delta_{0}\right) \min \left\{\delta_{k}, \psi\left(x_{k}, \delta_{0}\right) /\left(C \delta_{0}\right)\right\} .
$$

By Lemma 3.7, we may find $\beta$ and $\epsilon_{0}$ such that, for all $0<\epsilon \leqslant \epsilon_{0}$ and $x_{k} \in \operatorname{cl} B(\bar{x}, \epsilon)$,

$$
\psi\left(x_{k}, \delta_{0}\right) \geqslant \psi\left(x_{k}, \delta_{*}\right) \geqslant \beta>0 .
$$

Set $I_{1}=\left\{k \in I_{0}: \delta_{k} \geqslant \psi\left(x_{k}, \delta_{0}\right) / C \delta_{0}\right\}$ and $I_{2}=I_{0} \backslash I_{1}$. Then (3.13) shows that

$$
\sum_{k \in I_{1}}\left(\psi\left(x_{k}, \delta_{0}\right)\right)^{2}<+\infty
$$


and

$$
\sum_{k \in I_{2}} \psi\left(x_{k}, \delta_{0}\right) \delta_{k}<+\infty
$$

Hence, we may assume that there is $N(\epsilon)>0$ such that, for all $k \geqslant N(\epsilon)$ and $k \in I_{*}$, we have

$$
\operatorname{dist}\left(x_{k}, \bar{x}\right) \leqslant \frac{\epsilon}{2} \quad \text { and } \quad \sum_{k \in I_{2}, k \geqslant N(\epsilon)} \psi\left(x_{k}, \delta_{0}\right) \delta_{k} \leqslant \frac{\epsilon}{2} .
$$

By (3.17) and (3.14), we can prove that, for all $k$ with $k_{0}<k \leqslant k_{1}$, where $k_{0}$ is an index in $I_{*}$ with $k_{0} \geqslant N(\epsilon)$ and $k_{1}$ is the first index in $I_{1}$ greater than $k_{0}$,

$$
\begin{aligned}
\operatorname{dist}\left(x_{k}, \bar{x}\right) & \leqslant \operatorname{dist}\left(x_{k-1}, x_{k}\right)+\operatorname{dist}\left(x_{k-1}, \bar{x}\right) \\
& \leqslant \operatorname{dist}\left(x_{k_{0}}, \bar{x}\right)+\sum_{k_{0} \leqslant m<k, m \in I_{2}} \operatorname{dist}\left(x_{m+1}, x_{m}\right) \\
& \leqslant \operatorname{dist}\left(x_{k_{0}}, \bar{x}\right)+\sum_{k_{0} \leqslant m<k, m \in I_{2}} \operatorname{dist}\left(\exp _{x_{m}}\left(d_{m}\right), x_{m}\right) \\
& \leqslant \frac{1}{2} \epsilon+\sum_{k_{0} \leqslant m<k, m \in I_{2}} \delta_{m} \\
& \leqslant \epsilon,
\end{aligned}
$$

and therefore

$$
\psi\left(x_{k}, \delta_{0}\right) \geqslant \psi\left(x_{k}, \delta_{*}\right) \geqslant \beta>0 .
$$

This means that $I_{1}$ must be finite since otherwise $\sum_{k \in I_{1}}\left(\psi\left(x_{k}, \delta_{0}\right)\right)^{2}$ is not finite. Therefore, we have $\lim _{k \rightarrow \infty} x_{k}=\bar{x}$ for $k \in I_{0}$ and $\sum_{k \in I_{0}} \delta_{k}<\infty$. This contradicts the fact that $\delta_{k+1} \geqslant \delta_{k}$ for all large $k \in I_{0}$ and the proof is complete.

\section{A suitable model function}

In the previous Section 3, we have developed a general trust region method and established global convergence, provided that some criteria on the function $\Phi$ and the sequence $\left(B_{k}\right)_{k}$ hold true. The present section presents several choices for $\Phi$ which lead to convergent algorithms.

\subsection{The Clarke generalized directional derivative}

If $f$ were a smooth function, then clearly the function $\Phi$ would simply be the directional derivative of $f$. It is clear that since the function is not necessarily differentiable, we cannot use the differential of the objective function. However, we might be able to use generalized directional derivatives instead. Let us continue with the definition of the Clarke generalized directional derivative for locally Lipschitz functions on Riemannian manifolds; see Hosseini \& Pouryayevali (2011, 2013b).

Definition 4.1 (Clarke generalized directional derivative) Suppose $f: M \rightarrow \mathbb{R}$ is a locally Lipschitz function on a Riemannian manifold $M$. Let $\phi_{x}: U_{x} \rightarrow T_{x} M$ be an exponential chart at $x$. Given another point $y \in U_{x}$, consider $\sigma_{y, v}(t):=\phi_{y}^{-1}(t w)$, a geodesic passing through $y$ with derivative $w$, where $\left(\phi_{y}, y\right)$ 
is an exponential chart around $y$ and $d\left(\phi_{x} \circ \phi_{y}^{-1}\right)\left(0_{y}\right)(w)=v$. Then, the Clarke generalized directional derivative of $f$ at $x \in M$ in the direction $v \in T_{x} M$, denoted by $f^{\circ}(x ; v)$, is defined as

$$
f^{\circ}(x, v)=\limsup _{y \rightarrow x, t \downarrow 0} \frac{f\left(\sigma_{y, v}(t)\right)-f(y)}{t} .
$$

If $f$ is differentiable in $x \in M$, we define the gradient of $f$ as the unique vector $\operatorname{grad} f(x) \in T_{x} M$, which satisfies

$$
\langle\operatorname{grad} f(x), \xi\rangle=d f(x)(\xi) \quad \text { for all } \xi \in T_{x} M .
$$

Definition 4.2 (Subdifferential) We define the subdifferential of $f$, denoted by $\partial f(x)$, as the subset of $T_{x} M$ whose support function is $f^{\circ}(x ; \cdot)$. It can be proved (Hosseini \& Pouryayevali, 2011) that

$$
\partial f(x)=\operatorname{conv}\left\{\lim _{i \rightarrow \infty} \operatorname{grad} f\left(x_{i}\right):\left\{x_{i}\right\} \subseteq \Omega_{f}, x_{i} \rightarrow x\right\},
$$

where $\Omega_{f}$ is a dense subset of $M$ on which $f$ is differentiable. ${ }^{1}$

Therefore, we have a good candidate for the function $\Phi: T M \rightarrow \mathbb{R}$, which can be defined by

$$
\Phi(x, d):=f^{\circ}(x, d)=\sup \{\langle\xi, d\rangle: \xi \in \partial f(x)\} ;
$$

see Hosseini \& Pouryayevali (2011).

The resulting model function leads to a convergent algorithm as the following result shows.

Theorem 4.3 Let $f: M \rightarrow \mathbb{R}$ be locally Lipschitz and define $\Phi$ as in (4.1). Then, the function $\Phi$ satisfies Assumption 3.5. In particular, Algorithm 1 converges globally to a critical point of $f$.

Proof. The fact that $\Phi$ satisfies Assumption 3.5 follows directly from Theorems 2.4 and 2.9 of Hosseini $\&$ Pouryayevali (2011). Therefore, by Theorem 3.9 the resulting trust region algorithm converges.

\subsection{The $\varepsilon$-subdifferential}

A crucial observation is that the computation of $\Phi$ as in (4.1) can be impractical in case that no explicit expression for the subdifferential $\partial f(x)$ is available. Using an approximation of the Clarke subdifferential, we overcome this problem and define a local model that is practically and efficiently implementable.

The following definition presents an approximation of the subdifferential which can be computed approximately; see Grohs \& Hosseini (2015).

Definition 4.4 ( $\varepsilon$-subdifferential) Let $f: M \rightarrow \mathbb{R}$ be a locally Lipschitz function on a Riemannian manifold $M, \varepsilon<i_{M}(x)$. We define the $\varepsilon$-subdifferential of $f$ at $x$ denoted by $\partial_{\varepsilon} f(x)$ as follows;

$$
\partial_{\varepsilon} f(x)=\operatorname{conv}\left\{d \exp _{x}^{-1}(y)(\partial f(y)): y \in \operatorname{cl} B(x, \varepsilon)\right\} .
$$

The following result has been proved in Grohs et al. (2014).

LEMMA 4.5 Let $U$ be a compact subset of $M$ and $\varepsilon<i(U)$; then, for every open neighbourhood $W$ in $U$, the set-valued mapping $\partial_{\varepsilon} f: W \rightarrow T M$ is upper semicontinuous.

\footnotetext{
${ }^{1}$ Note that $\lim \operatorname{grad} f\left(x_{i}\right)$ in this definition is obtained as follows. Let $\xi_{i} \in T_{x_{i}} M, i=1,2, \ldots$ be a sequence of tangent vectors of $M$ and $\xi \in T_{X} M$. We say that $\xi_{i}$ converges to $\xi$, denoted by $\lim \xi_{i}=\xi$, provided that $x_{i} \rightarrow x$ and, for any smooth vector field $X$, $\left\langle\xi_{i}, X\left(x_{i}\right)\right\rangle \rightarrow\langle\xi, X(x)\rangle$.
} 
Now we construct a suitable model function for the following unconstrained optimization problem,

$$
\min _{x \in M} f(x)
$$

where $f: M \rightarrow \mathbb{R}$ is a locally Lipschitz function. Assume that $D$ is a bounded open subset of $M$ and $\varepsilon<i(\operatorname{cl}(D))$. We define $\Phi: T D \rightarrow \mathbb{R}$ by

$$
\Phi(x, d):=\sup \left\{\langle\xi, d\rangle: \xi \in \partial_{\varepsilon} f(x)\right\}, \quad \text { for every } x \in D .
$$

We now show that this function $\Phi$ satisfies Assumption 3.5.

Theorem 4.6 The function $\Phi: T D \rightarrow \mathbb{R}$ defined by (4.2) satisfies Assumption 3.5.

Proof. It is easy to prove that $\Phi$ satisfies Assumptions (3.6) and (3.8). Moreover, $\left.\Phi\right|_{T_{x} M}$ is upper semicontinuous; to show this for every $\epsilon>0$, we set $\delta<\epsilon / K$, where $K$ is the Lipschitz constant of $f$ on a neighbourhood of $x$. Now, if $\|d-w\|<\delta$, then

$$
\Phi(x, w) \leqslant \Phi(x, w-d)+\Phi(x, d) \leqslant K\|w-d\|+\Phi(x, d)<\epsilon+\Phi(x, d),
$$

therefore the claim is proved.

To prove that $\Phi$ satisfies Assumption (3.9), note that, for $(x, d) \in T M$,

$$
f\left(\exp _{x}(d)\right)-f(x) \leqslant f^{\circ}(x, d)+o(\|d\|) \leqslant \Phi(x, d)+o(\|d\|) .
$$

Now, we prove that $\Phi$ satisfies Assumption (3.10). To this end, we first prove that: for each $\epsilon>0$, there exists $\delta>0$ such that, for $x \in B\left(x_{0}, \delta\right) \subset D$,

$$
L_{x x_{0}}\left(\partial_{\varepsilon} f(x)\right) \subseteq \partial_{\varepsilon} f\left(x_{0}\right)+\epsilon B_{T_{x_{0}} M},
$$

where $B_{T_{x_{0}} M}$ is the unit ball of $T_{x_{0}} M$. To see this, note that, for $\epsilon>0$, the set $\partial_{\varepsilon} f\left(x_{0}\right)+\epsilon B_{T_{x_{0}} M}$ is an open neighbourhood of $L_{x_{0} x_{0}}\left(\partial_{\varepsilon} f\left(x_{0}\right)\right)=\partial_{\varepsilon} f\left(x_{0}\right)$. It follows from the continuity of (2.1) that there exists an open neighbourhood $V \subset T M$ of $\partial_{\varepsilon} f\left(x_{0}\right)$ such that

$$
C(V) \subseteq \partial_{\varepsilon} f\left(x_{0}\right)+\epsilon B_{T_{x_{0}} M} .
$$

By the upper semicontinuity of $\partial_{\varepsilon} f$, there exists a neighbourhood $V^{\prime}$ of $x_{0}$ such that, for each $x \in V^{\prime}$, we have $\partial_{\varepsilon} f(x) \subseteq V$. Now, let $x \in V^{\prime}$; then

$$
L_{x x_{0}}\left(\partial_{\varepsilon} f(x)\right) \subseteq \partial_{\varepsilon} f\left(x_{0}\right)+\epsilon B_{T_{x_{0}} M},
$$

as required. Now, we claim that there exists $\delta_{*}>0$ such that, for all $x \in D$ and $d_{x} \in T_{x} M$ with $\left\|d_{x}\right\|<\delta_{*}$, we have that $\Phi$ is upper semicontinuous at $d_{x}$.

To prove the claim; since $l_{x}(y):=d \exp _{x}^{-1}(y)$ is a smooth function with respect to $y$, we have that it is bounded on $\operatorname{cl} B(x, \varepsilon)$ by some $m_{x} \geqslant 0$, from the Lipschitzness of $f$ on $\operatorname{cl} B(x, \varepsilon)$, Theorem 2.9 of Hosseini \& Pouryayevali (2011) implies that, for every $\xi \in \partial_{\varepsilon} f(x),\|\xi\| \leqslant m_{x} K_{x}$. Since cl(D) is compact, there exists a finite number of neighbourhoods $B\left(x_{i}, \varepsilon\right)$ such that $D \subset \bigcup_{i=1}^{n} B\left(x_{i}, \varepsilon\right)$. Assume that $m_{1} K:=$ $\min \left\{m_{x_{i}} K_{x_{i}}: i=1, \ldots, n\right\}, \delta_{*}:=m_{1} K$. Let $x$ and $d_{x}$ be, respectively, arbitrary elements of $D$ and $T_{x} M$ with $\left\|d_{x}\right\|<\delta_{*}$. 
We prove that, for each $\epsilon>0$, there exists $\delta>0$ such that

$$
\Phi\left(y, d_{y}\right)<\Phi\left(x, d_{x}\right)+\epsilon, \text { provided } \operatorname{dist}_{T M}\left(d_{x}, d_{y}\right)<\delta
$$

Assume that $0<\epsilon<1$; then, there exists $\delta_{1}>0$ such that, for each $y \in B\left(x, \delta_{1}\right) \subset D$,

$$
L_{y x}\left(\partial_{\varepsilon} f(y)\right) \subseteq \partial_{\varepsilon} f(x)+\frac{\epsilon}{3 K m_{1}} B_{T_{x} M} .
$$

Since $\left.\Phi\right|_{T_{x} M}$ is upper semicontinuous and $\Phi\left(x, 0_{x}\right)=0$, hence there exists $\delta_{3}>0$ such that $\left\|w_{x}\right\|<\delta_{3}$ implies that $\Phi\left(x, w_{x}\right)<\epsilon / 3$. Assume that $\sigma:=\min \left\{\delta_{3}, m_{1} K \epsilon / 3\right\}$; then the continuity of (2.1) implies that there exists $\delta_{2}>0$,

$$
\left\|L_{y x}\left(d_{y}\right)-d_{x}\right\|<\sigma, \text { provided } \operatorname{dist}_{T M}\left(d_{x}, d_{y}\right)<\delta_{2} .
$$

Let $\delta<\min \left\{\delta_{1}, \delta_{2}, \delta_{3}\right\}$ be such that $B(x, \delta)$ is convex. We suppose that $\operatorname{dist}_{T M}\left(d_{x}, d_{y}\right)<\delta$; then $\operatorname{dist}(x, y)<\delta$ and $y \in B(x, \delta) \subset D$. Therefore,

$$
\begin{aligned}
\Phi\left(y, d_{y}\right) & =\sup _{\xi}\left\{\left\langle\xi, d_{y}\right\rangle: \xi \in \partial_{\varepsilon} f(y)\right\} \\
& =\sup \left\{\left\langle L_{y x} \xi, L_{y x} d_{y}\right\rangle: \xi \in \partial_{\varepsilon} f(y)\right\} \\
& \leqslant \sup _{\eta}\left\{\left\langle\eta, L_{y x} d_{y}\right\rangle: \eta \in \partial_{\varepsilon} f(x)+\frac{\epsilon}{3 K m_{1}} B_{T_{x} M}\right\} \\
& \leqslant \sup _{\theta, v}\left\{\left\langle\theta+v, d_{x}+w_{x}\right\rangle: \theta \in \partial_{\varepsilon} f(x), v \in \frac{\epsilon}{3 K m_{1}} B_{T_{x} M},\left\|w_{x}\right\|<\sigma\right\} \\
& \leqslant \sup _{\theta}\left\{\left\langle\theta, d_{x}\right\rangle+\left\langle\theta, w_{x}\right\rangle: \theta \in \partial_{\varepsilon} f(x),\left\|w_{x}\right\|<\sigma\right\}+\frac{\epsilon^{2}}{9}+\frac{\epsilon}{3} \\
& \leqslant \Phi\left(x, d_{x}\right)+\frac{\epsilon^{2}}{9}+\frac{\epsilon}{3}+\Phi\left(x, w_{x}\right) \leqslant \Phi\left(x, d_{x}\right)+\epsilon .
\end{aligned}
$$

It is worth mentioning that every critical point with respect to $\Phi$ defined by (4.2) is an $\varepsilon$-stationary point; i.e., there is $y$ in $\operatorname{cl} B(x, \varepsilon)$ such that $0 \in \partial f(y)$. A key property of the $\varepsilon$-subdifferential is that it can be approximated efficiently. In our implementations, we substitute the $\varepsilon$-subdifferential of the objective function $f$ with its approximation presented in Grohs et al. (2014). Indeed, to approximate the $\varepsilon$-subdifferential at $x_{k}$, we start with the gradient of an arbitrary point nearby $x_{k}$, and move the gradient to the tangent space in $x_{k}$ via the derivative of the logarithm mapping, and in every subsequent iteration, the gradient of a new point nearby $x_{k}$ is computed and moved to the tangent space in $x_{k}$ to add to the working set to improve the approximation of $\partial_{\varepsilon} f\left(x_{k}\right)$. Indeed, we do not want to provide a description of the entire $\varepsilon$-subdifferential set at each iteration; what we do is approximate $\partial_{\varepsilon} f\left(x_{k}\right)$ by the convex hull of its elements. In this way let $W_{l}:=\left\{v_{1}, \ldots, v_{l}\right\} \subseteq \partial_{\varepsilon} f\left(x_{k}\right)$; then, we define

$$
w_{l}:=\underset{v \in \operatorname{conv} W_{l}}{\operatorname{argmin}}\|v\|
$$


Now, if we have

$$
f\left(\exp _{x_{k}}\left(\varepsilon g_{l}\right)\right)-f\left(x_{k}\right) \leqslant-c \varepsilon\left\|w_{l}\right\|, \quad c \in(0,1),
$$

where $g_{l}=-w_{l} /\left\|w_{l}\right\|$; then, we can say conv $W_{l}$ is an acceptable approximation for $\partial_{\varepsilon} f\left(x_{k}\right)$. Otherwise, we add a new element of $\partial_{\varepsilon} f\left(x_{k}\right) \backslash$ conv $W_{l}$ to $W_{l}$. Indeed, having (4.4) implies that the set conv $W_{l}$ contains a vector $w_{l}$ such that $g_{l}=-w_{l} /\left\|w_{l}\right\|$ is a good approximation of the steepest descent direction. See Grohs et al. (2014) for further details on how to algorithmically realize this approximation procedure. The following lemma proves that if $W_{l}$ is not an acceptable approximation for $\partial_{\varepsilon} f(x)$, then there exists $v_{l+1} \in \partial_{\varepsilon} f(x)$ such that $\left\langle v_{l+1}, g_{l}\right\rangle \geqslant-c\left\|w_{l}\right\|>-\left\|w_{l}\right\|$, therefore $v_{l+1} \in \partial_{\varepsilon} f(x) \backslash \operatorname{conv} W_{l}$; for a proof see Grohs et al. (2014).

Lemma 4.7 Let $W_{l}=\left\{v_{1}, \ldots, v_{l}\right\} \subset \partial_{\varepsilon} f(x), 0 \notin \operatorname{conv} W_{l}$ and

$$
w_{l}=\operatorname{argmin}\left\{\|v\|: v \in \operatorname{conv} W_{l}\right\} .
$$

If we have $f\left(\exp _{x}\left(\varepsilon g_{l}\right)\right)-f(x)>-c \varepsilon\left\|w_{l}\right\|$, where $g_{l}=-w_{l} /\left\|w_{l}\right\|$, then there exist $\theta_{0} \in(0, \varepsilon]$ and $\bar{v}_{l+1} \in$ $\partial f\left(\exp _{x}\left(\theta_{0} g_{l}\right)\right)$ such that

$$
\left\langle d \exp _{x}^{-1}\left(\exp _{x}\left(\theta_{0} g_{l}\right)\right)\left(\bar{v}_{l+1}\right), g_{l}\right\rangle \geqslant-c\left\|w_{l}\right\|,
$$

and $v_{l+1}:=d \exp _{x}^{-1}\left(\exp _{x}\left(\theta_{0} g_{l}\right)\right)\left(\bar{v}_{l+1}\right) \notin \operatorname{conv} W_{l}$.

Algorithm 2 is used to find a vector $v_{l+1} \in \partial_{\varepsilon} f(x)$ which can be added to the set $W_{l}$ in order to improve the approximation of $\partial_{\varepsilon} f(x)$. It is easy to prove by Mahdavi-Amiri \& Yousefpour (2012, Propositions 3.2 and 3.3) that this algorithm terminates after finitely many iterations.

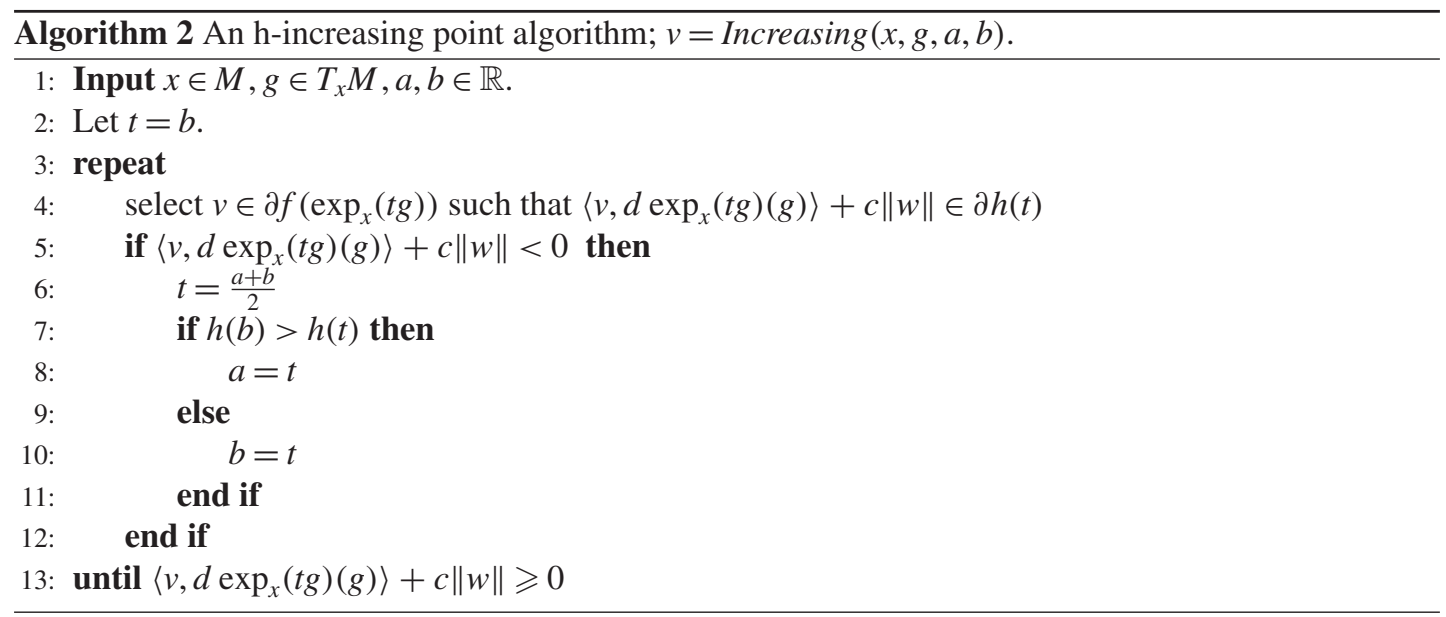

Therefore, using an approximation of $\partial_{\varepsilon} f\left(x_{k}\right)$ we define a function $\Phi\left(x_{k}, d\right):=\max \{\langle\xi, d\rangle: \xi \in$ conv $\left.W_{l}\right\}$, which approximately satisfies our assumptions and is easily computable at every $d \in T_{x_{k}} M$. Indeed, if we assume that $i \in\{1, \ldots, l\}$ is such that, for a fixed $d \in T_{x_{k}} M$, we have $\left\langle v_{j}, d\right\rangle \leqslant\left\langle v_{i}, d\right\rangle$ for every $j \in\{1, \ldots, l\}$, then, for every $\xi \in \operatorname{conv} W_{l}$, we have $\xi:=\sum_{s=1}^{l} \alpha_{s} v_{s}$ such that $\sum_{s=1}^{l} \alpha_{s}=1$ and therefore $\langle\xi, d\rangle \leqslant\left\langle v_{i}, d\right\rangle$. 


\section{Numerical experiments}

The Riemannian nonsmooth trust region algorithm presented in the previous section was implemented in MATLAB. To the best of our knowledge, our algorithm is the first practical nonsmooth trust region algorithm for locally Lipschitz functions defined on Riemannian manifolds. In our implementation, we update the sequence of matrices $\left\{B_{k}\right\}$ by using the BFGS method; see Qi et al. (2010). Moreover, we compare the nonsmooth trust region algorithm with Riemannian subgradient descent algorithms presented in Borckmans et al. (2014), Grohs \& Hosseini (2015) and Dirr et al. (2007). The number of function evaluations is used as a measure efficiency for the algorithms. The parameters are initialized similar to the smooth version of the classical trust method. We set the parameters as $c_{1}=0.75, c_{2}=0$, $c_{3}=0.5, c_{4}=2, c=10^{-4}, \varepsilon=10^{-6}, \delta=10^{-1}, \delta_{1}=10^{-1}$.

The unit sphere $S^{2}$ is the smooth compact manifold

$$
S^{2}=\left\{x \in \mathbb{R}^{3}:\|x\|=1\right\},
$$

and the global coordinates on $S^{2}$ are naturally given by this embedding into $\mathbb{R}^{3}$. The tangent space at a point $x \in S^{2}$ is

$$
T_{x} S^{2}=\left\{v \in \mathbb{R}^{3}:\langle x, v\rangle=0\right\}
$$

The inner product on $T_{x} S^{2}$ is defined by

$$
\langle v, w\rangle_{T_{x} S^{2}}=\langle v, w\rangle_{\mathbb{R}^{3}}
$$

The exponential map

$$
\exp _{x}: T_{x} S^{2} \rightarrow S^{2}
$$

is defined by

$$
\exp _{x}(v)=\cos (\|v\|) x+\sin (\|v\|) \frac{v}{\|v\|}
$$

Moreover, if $x \in S^{2}$, then

$$
\exp _{x}^{-1}: S^{2} \rightarrow T_{x} S^{2}
$$

is defined by

$$
\exp _{x}^{-1}(y)=\frac{\theta}{\sin (\theta)}(y-x \cos (\theta)),
$$

where $\theta=\arccos \langle x, y\rangle$. The Riemannian distance between two points $x, y$ in $S^{2}$ is given by

$$
\operatorname{dist}(x, y)=\arccos \langle x, y\rangle \text {. }
$$

Let $t \rightarrow \gamma(t)$ be a geodesic on $S^{2}$ and let $u=\gamma^{\circ}(0) /\left\|\gamma^{\circ}(0)\right\|$. The parallel translation of a vector $v \in T_{\gamma(0)} S^{2}$, along the geodesic $\gamma$, is given by Absil et al. (2008):

$$
L_{\gamma(0) \gamma(t)}(v)=-\gamma(0) \sin \left(\left\|\gamma^{\circ}(0)\right\| t\right) u^{\prime} v+u \cos \left(\left\|\gamma^{\circ}(0)\right\| t\right) u^{\prime} v+\left(I-u u^{\prime}\right) v .
$$




\subsection{Denoising on a sphere}

First, we are going to solve the one-dimensional total variation problem for functions which map into a two-dimensional sphere $S^{2}$. Assuming that $M$ is a manifold, consider the minimization problem

$$
\min _{u \in B V([0,1] ; M)}\left\{F(u):=\operatorname{dist}_{2}(f, u)^{2}+\lambda\|\nabla u\|_{1}\right\},
$$

where $f:[0,1] \rightarrow M$ is the given (noisy) function, $u$ is a function of bounded variation from [0,1] to $M$, dist ${ }_{2}$ is the distance on the function space $L^{2}([0,1] ; M)$ and $\lambda>0$ is a Lagrangian parameter (Rudin et al., 1992). Note that, for every $w \in[0,1], \nabla u(w): \mathbb{R} \rightarrow T_{u(w)} M$ and $\|\nabla u\|_{1}=\int_{[0,1]}\|\nabla u(w)\| \mathrm{d} w$. Now, we can formulate a discrete version of the problem (5.1) by restricting the space of functions to $V_{h}^{M}$, which is the space of all geodesic finite element functions for $M$ associated with a regular grid on [0,1]; see Sander (2010) and Grohs et al. (2014). We refer the reader to Sander (2010) for the definition of geodesic finite element spaces $V_{h}^{M}$.

Using the nodal evaluation operator $\varepsilon: V_{h}^{M} \rightarrow M^{n},\left(\varepsilon\left(v_{h}\right)\right)_{i}=v_{h}\left(x_{i}\right)$, where $x_{i}$ is the $i$ th vertex of the simplicial grid on $[0,1]$, one can find an equivalent problem defined on $M^{n}$ as follows:

$$
\min _{u \in M^{n}}\left\{F_{*}(u):=\operatorname{dist}_{*}(\varepsilon(f), u)^{2}+\lambda\left\|\nabla\left(\varepsilon^{-1}(u)\right)\right\|_{1}\right\},
$$

where dist ${ }_{*}$ is the Riemannian distance on $M^{n}$.

Let $\varepsilon(f)=\left(p_{1}, \ldots, p_{n}\right)$; then $F_{*}: M^{n} \rightarrow \mathbb{R}$ can be defined by

$$
F_{*}\left(u_{1}, \ldots, u_{n}\right)=\Sigma_{i=1}^{n} \operatorname{dist}\left(p_{i}, u_{i}\right)^{2}+\lambda \Sigma_{i=1}^{n-1} \operatorname{dist}\left(u_{i}, u_{i+1}\right),
$$

where dist is the Riemannian distance on $M$.

Now, we assume that $M=S^{2}$. First, we need to define a function from $[0,1]$ to $S^{2}$ to get the original image. Afterwards, we add a gaussian noise to the image to get the noisy image. Finally, we apply Algorithm 1 to the function $F_{*}$ defined on $M^{100}$ to get the denoised image; see Fig. 1.

Table 1 provides the numerical results for the TV regularization on $S^{2}$ using the nonsmooth trust region method and $\varepsilon$-subdifferential method. In this table, 'nfval' stands for the number of function evaluations; we also have presented the minimum value of the function. The number of function evaluations is considered as a measure of efficiency for the trust region method.

\subsection{Denoising on $P(2)$}

Data taking values in a manifold appear naturally in various signal and image processing applications. One example is diffusion tensor imaging, where the data live in the Riemannian manifold of positive (definite) matrices; see Basser et al. (1994), Pennec et al. (2006) and Weinmann et al. (2014). The space of all $n \times n$ symmetric, positive definite matrices will be denoted by $P(n)$. The tangent space to $P(n)$ at any of its points $P$ is the space $T_{P} P(n)=\{P\} \times S(n)$, where $S(n)$ is the space of symmetric $n \times n$ matrices. On each tangent space $T_{P} P(n)$, the inner product is defined by

$$
\langle A, B\rangle_{T_{P} P(n)}=\operatorname{tr}\left(P^{-1} A P^{-1} B\right) .
$$

The Riemannian distance between $P, Q \in P(n)$ is given by

$$
\operatorname{dist}(P, Q)=\left(\Sigma_{i=1}^{n} \ln ^{2}\left(\lambda_{i}\right)\right)^{(1 / 2)},
$$




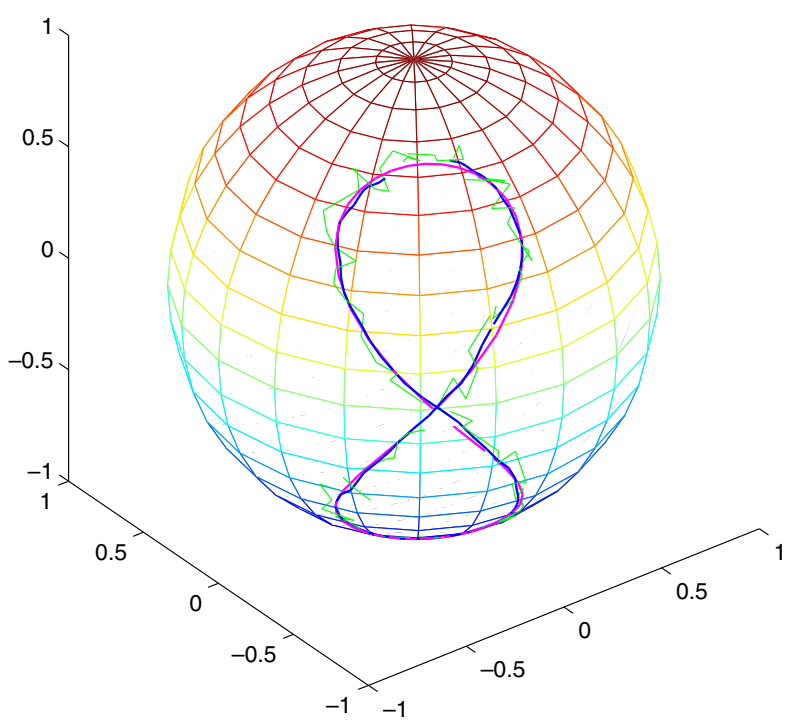

FIG. 1. TV regularization on $S^{2}$.

TABLE 1 Numerical results in terms of number of function evaluations and the final obtained value of the function for TV regularization

\begin{tabular}{lcccr}
\hline No. & $f$ in trust region method & nfeval & $f$ in $\varepsilon$-subdifferential method & nfeval \\
\hline 1 & 3.2266 & 365 & 3.2362 & 934 \\
2 & 3.2512 & 327 & 3.2579 & 976 \\
3 & 3.2902 & 424 & 3.3145 & 3316 \\
4 & 3.2794 & 509 & 3.2896 & 3097 \\
5 & 3.2366 & 365 & 3.2472 & 934 \\
6 & 3.2212 & 327 & 3.2572 & 971 \\
7 & 3.2432 & 424 & 3.3066 & 3216 \\
8 & 3.2884 & 509 & 3.2976 & 1097 \\
9 & 3.2187 & 325 & 3.2472 & 1100 \\
10 & 3.2012 & 327 & 3.2972 & 1906 \\
11 & 3.2302 & 424 & 3.3136 & 1001 \\
12 & 3.2394 & 509 & 3.2576 & 1097 \\
13 & 3.2234 & 365 & 3.2365 & 4934 \\
14 & 3.2456 & 327 & 3.2542 & 3976 \\
15 & 3.2912 & 424 & 3.3298 & 3316 \\
\hline
\end{tabular}

where $\lambda_{i}, i=1, \ldots, n$, are eigenvalues of $P^{-1} Q$. The exponential map

$$
\exp _{P}: S(n) \rightarrow P(n)
$$

is defined by

$$
\exp _{P}(v)=P^{1 / 2} \exp \left(P^{-1 / 2} v P^{-1 / 2}\right) P^{1 / 2}
$$



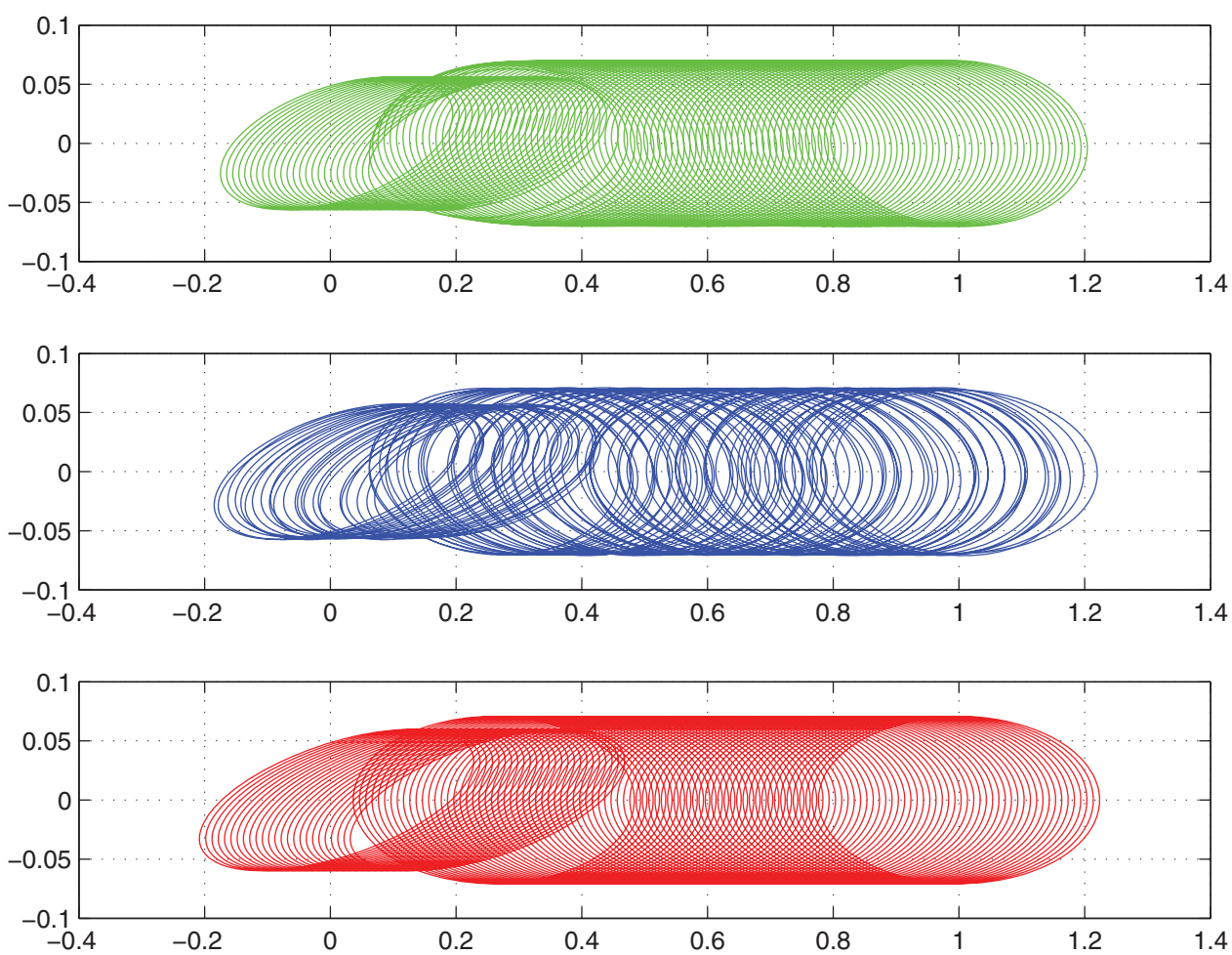

FIG. 2. TV regularization on $P(2)$. Down-to-up: the original image, the noisy image and the denoised image.

Moreover, if $P \in P(n)$, then

$$
\exp _{P}^{-1}: P(n) \rightarrow S(n)
$$

is defined by

$$
\exp _{P}^{-1}(Q)=P^{1 / 2} \log \left(P^{-1 / 2} Q P^{-1 / 2}\right) P^{1 / 2}
$$

where log and exp denote, respectively, the logarithm and exponential functions on matrix space. For another example, we assume that $M=P(2)$. We add a noise to an original image on $P(2)$. Then, we apply our nonsmooth trust region algorithm to $F_{*}$ on $M^{100}$ to denoise the noisy image. In Fig. 2, we present the results regarding the minimization of $F_{*}$ on $M^{100}$. Table 2 provides the numerical results for the TV regularization on $P(2)$ using the nonsmooth trust region method and the $\varepsilon$-subdifferential method.

\subsection{Riemannian geometric median on a sphere}

Our third numerical experiment is concerned with the Riemannian geometric median on $S^{2}$. Let $M$ be a Riemannian manifold. Given points $p_{1}, \ldots, p_{m}$ in $M$ and corresponding positive real weights 
TABLE 2 Numerical results in terms of number of function evaluations and the final obtained value of the function for TV regularization on $P(2)$

\begin{tabular}{lcccr}
\hline No. & $f$ in trust region method & nfeval & $f$ in $\varepsilon$-subdifferential method & nfeval \\
\hline 1 & 0.2340 & 180 & 0.2354 & 1344 \\
2 & 0.1981 & 111 & 0.1917 & 1541 \\
3 & 0.2102 & 102 & 0.2231 & 1681 \\
4 & 0.1623 & 154 & 0.1734 & 894 \\
5 & 0.1689 & 134 & 0.1670 & 800 \\
6 & 0.1890 & 159 & 0.1840 & 1680 \\
7 & 0.2301 & 451 & 0.2401 & 970 \\
8 & 0.1891 & 167 & 0.1871 & 1356 \\
9 & 0.1924 & 101 & 0.1981 & 1451 \\
10 & 0.2101 & 186 & 0.2191 & 1189 \\
11 & 0.1690 & 192 & 0.1673 & 891 \\
12 & 0.1871 & 171 & 0.1780 & 3911 \\
13 & 0.1988 & 161 & 0.1981 & 1601 \\
14 & 0.2121 & 195 & 0.2191 & 761 \\
15 & 0.2241 & 108 & 0.2240 & 1316 \\
\hline
\end{tabular}

$w_{1}, \ldots, w_{m}$, with $\sum_{i=1}^{m} w_{i}=1$, define the weighted sum of distance functions

$$
f(q)=\sum_{i=1}^{m} w_{i} \operatorname{dist}\left(p_{i}, q\right),
$$

where dist is the Riemannian distance function on $M$. We define the weighted geometric median $x$ as the minimizer of $f$. When all the weights are equal, $w_{i}=1 / m$, we call $x$ simply the geometric median. Now, we assume that $M=S^{2}$.

Table 3 provides the numerical results for finding the geometric median on $S^{2}$ with $m=5000$, using the nonsmooth trust region method and the $\varepsilon$-subdifferential method. The starting point and the points $p_{i}$ are chosen randomly. As before, 'nfval' stands for the number of function evaluations; we also have presented the minimum value of the function. The number of function evaluations is considered as a measure of efficiency for the trust region method.

\subsection{Rayleigh quotients on a sphere}

Now, we are going to compare our nonsmooth trust region algorithm with the Riemannian subgradient descent presented in Dirr et al. (2007). To this end, we consider the maximum of $m$ Rayleigh quotients on the sphere $S^{n-1}$, i.e.,

$$
f(x)=\max _{i=1, \ldots, m} \frac{1}{2} x^{\prime} A_{i} x
$$

where $A_{i} \in \mathbb{R}^{n \times n}$ is symmetric. Our aim is to find a minimum of $f$. Table 4 provides the numerical results for finding the minimum of $f$ on $S^{2}$ with $m=20$, using the nonsmooth trust region method and the subgradient descent method. 
TABLE 3 Numerical results in terms of number of function evaluations and the final obtained value of the function for the geometric median on $S^{2}$

\begin{tabular}{lcrcr}
\hline No. & $f$ in trust region method & nfeval & $f$ in $\varepsilon$-subdifferential method & nfeval \\
\hline 1 & 0.4306 & 8 & 0.4365 & 192 \\
2 & 0.4302 & 11 & 0.4305 & 67 \\
3 & 0.4101 & 2 & 0.4200 & 108 \\
4 & 0.4338 & 13 & 0.4390 & 110 \\
5 & 0.4206 & 13 & 0.4273 & 93 \\
6 & 0.4312 & 5 & 0.4326 & 97 \\
7 & 0.4302 & 11 & 0.4321 & 105 \\
8 & 0.4394 & 11 & 0.4398 & 109 \\
9 & 0.4316 & 11 & 0.4321 & 98 \\
10 & 0.4212 & 8 & 0.4237 & 97 \\
11 & 0.4302 & 9 & 0.4388 & 128 \\
12 & 0.4334 & 10 & 0.4399 & 167 \\
13 & 0.4266 & 6 & 0.4280 & 211 \\
14 & 0.4292 & 5 & 0.4300 & 145 \\
15 & 0.4372 & 4 & 0.4384 & 316 \\
\hline
\end{tabular}

TABLE 4 Numerical results in terms of number of function evaluations and the final obtained value of the function for Rayleigh quotients on $S^{2}$

\begin{tabular}{lcccr}
\hline No. & $f$ in trust region method & nfeval & $f$ in subdifferential method & nfeval \\
\hline 1 & 0.6529 & 128 & 1.7832 & 501 \\
2 & 0.3294 & 116 & 0.3341 & 3614 \\
3 & 0.7321 & 116 & 0.7996 & 5501 \\
4 & 0.5211 & 123 & 0.5458 & 2123 \\
5 & 0.5156 & 117 & 0.4962 & 509 \\
6 & 0.6321 & 156 & 0.6578 & 1504 \\
7 & 0.4321 & 167 & 0.4567 & 1350 \\
8 & 0.6421 & 127 & 0.6432 & 680 \\
9 & 0.7691 & 117 & 0.7761 & 1384 \\
10 & 0.4021 & 116 & 0.4187 & 890 \\
11 & 0.6531 & 170 & 0.6520 & 1230 \\
12 & 0.5090 & 175 & 0.5021 & 2012 \\
13 & 0.5401 & 149 & 0.5721 & 1890 \\
14 & 0.3489 & 110 & 0.3901 & 2056 \\
15 & 0.3209 & 113 & 0.3009 & 1105 \\
\hline
\end{tabular}

Moreover, we say that an algorithm solves a problem successfully if the following condition is satisfied:

$$
\frac{\left|f_{\mathrm{opt}}-f *\right|}{\left|f_{\mathrm{opt}}+1\right|} \leqslant \epsilon
$$

where $f_{\text {opt }}$ is the minimum value of the function and $f *$ is the minimum value obtained by the algorithm, and $\epsilon=10^{-4}$ is an accuracy level. Figure 3 shows that the nonsmooth trust region algorithm solves the problem. 


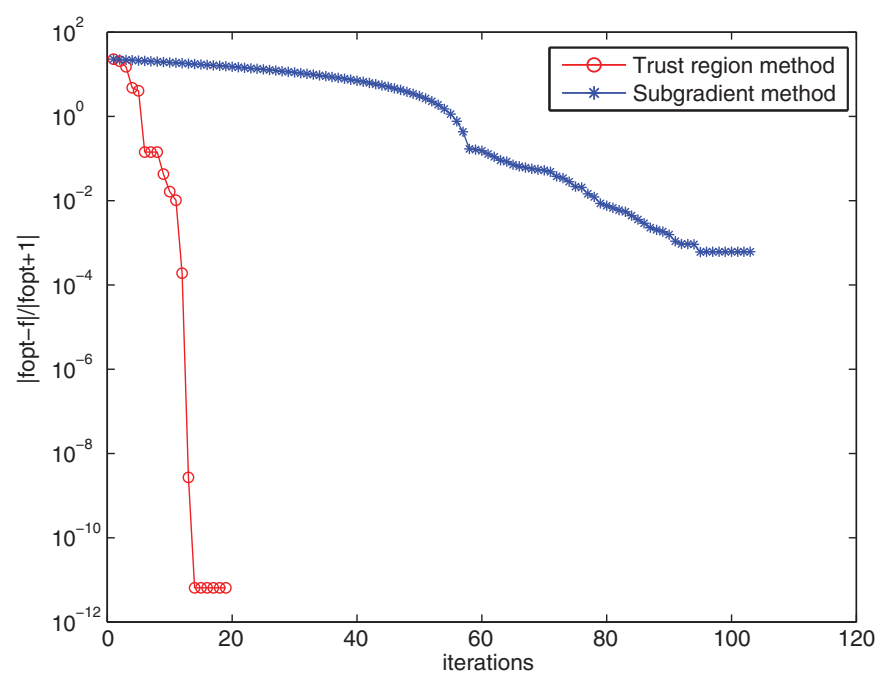

FIG. 3. Rayleigh quotients on $S^{2}$. We compare the trust region method introduced in this paper with the Riemannian subgradient algorithm from Dirr et al. (2007).

\subsection{Sphere packings on Grassmannians}

The sphere packings on Grassmannians have many applications in wireless communication and statistics, and seem to be good candidates for use in quantum information theory; see, for example, Ashikhmin \& Calderbank (2010) and Ozbek \& Ruyet (2013). We assume that the Grassmannian $\operatorname{Gr}(n, k)$ is the set of all $k$-dimensional linear subspaces of $\mathbb{R}^{n}$. In this section, we consider the problem of the packing of $m$ spherical balls on $\operatorname{Gr}(n, k)$ with respect to the chordal distance. Let $B(P, r)$ denote the ball in $\operatorname{Gr}(n, k)$ with respect to chordal distance. Then, we would like to find $m$ points $P_{1}, \ldots, P_{m}$ in $\operatorname{Gr}(n, k)$ such that

$$
\max \left\{r \mid \forall i \neq j: B\left(P_{i}, r\right) \cap B\left(P_{j}, r\right)=\emptyset\right\}
$$

is maximized. This problem has been solved in Dirr et al. (2007) using a subgradient method.

Indeed, $\operatorname{Gr}(n, k)$ can be identified with the set $\left\{P \in S(n) \mid P^{2}=P, \operatorname{tr}(P)=k\right\}$; see Helmke \& Moore (1994). Moreover, the tangent space of the Grassmannian at the point $P$, denoted by $T_{P} \operatorname{Gr}(n, k)$, is the following set:

$$
T_{P} \operatorname{Grass}(n, k)=\{P \Omega-\Omega P \mid \Omega \in \operatorname{so}(n)\},
$$

where

$$
\operatorname{so}(n)=\left\{\Omega \in \mathbb{R}^{n \times n} \mid \Omega^{\prime}=-\Omega\right\} .
$$

As $\operatorname{Gr}(n, k)$ is a subset of the Euclidean vector space $S(n)$, the scalar product $\langle P, Q\rangle:=\operatorname{tr}(P Q)$ induces a Riemannian metric on it. Therefore, the chordal distance on $\operatorname{Gr}(n, k)$, denoted by $\operatorname{dist}(P, Q)$, is defined by

$$
\operatorname{dist}(P, Q)=\sqrt{\frac{1}{2}}\|P-Q\|_{F},
$$


TABLE 5 Numerical results in terms of number of function evaluations and the final obtained value of the function for sphere packings on Grassmannians

\begin{tabular}{lcccr}
\hline No. & Minimal distance in TR & nfeval & Minimal distance in SB & nfeval \\
\hline 1 & 1.851 & 167 & 1.706 & 601 \\
2 & 1.689 & 104 & 1.674 & 231 \\
3 & 1.795 & 115 & 1.779 & 1109 \\
4 & 1.862 & 146 & 1.796 & 1123 \\
5 & 1.801 & 110 & 1.892 & 234 \\
6 & 1.813 & 156 & 1.711 & 1609 \\
7 & 1.862 & 145 & 1.791 & 1209 \\
8 & 1.874 & 132 & 1.776 & 890 \\
9 & 1.719 & 112 & 1.721 & 1409 \\
10 & 1.789 & 123 & 1.799 & 980 \\
11 & 1.708 & 145 & 1.769 & 430 \\
12 & 1.804 & 154 & 1.704 & 1230 \\
13 & 1.897 & 179 & 1.791 & 1679 \\
14 & 1.676 & 134 & 1.659 & 2340 \\
15 & 1.631 & 104 & 1.611 & 1306 \\
\hline
\end{tabular}

where $\|\cdot\|_{F}$ denotes the Frobenius norm. On $\operatorname{Gr}(n, k)$ with the induced Riemannian metric, the geodesic $\gamma$ emanating from $P$ in the direction $\eta \in T_{P} \operatorname{Grass}(n, k)$ is defined by

$$
\gamma(t)=\exp (t(\eta P-P \eta)) P \exp (-t(\eta P-P \eta))
$$

The problem (5.4) is equivalent to minimizing the following nonsmooth function

$$
F\left(P_{1}, \ldots, P_{m}\right):=\max _{i \neq j} \operatorname{tr}\left(P_{i} P_{j}\right)
$$

on $\operatorname{Gr}(n, k) \times \cdots \times \operatorname{Gr}(n, k)$; see Lageman \& Helmke (2007).

In Table 5, we illustrate the results of the nonsmooth subgradient (SB) method and nonsmooth trust region method (TR) for the sphere packing in $\operatorname{Gr}(16,2)$ with $m=10$ and the same arbitrary starting points for both methods.

\section{Conclusions}

We have presented a practical algorithm in the context of trust region methods for nonsmooth problems on Riemannian manifolds. To the best of our knowledge, this is the first paper on the nonsmooth trust region method on Riemannian manifolds. We also introduce a practical local model in our trust region scheme for locally Lipschitz functions. We have seen that the use of an exponential map yields trust region subproblems expressed in Euclidean spaces $T_{x} M$. Therefore, all the classical methods for solving the trust region subproblem can be applied. In our implementation, we use the approach based on the Cauchy point and the CG-Steihaug methods; see Akbari et al. (2015). The main result is the global convergence property of our trust region method which is stated in Theorem 3.9.

An implementation of our proposed trust region algorithm, along with the subgradient and $\varepsilon$-subgradient methods, is given in the MATLAB environment and tested on some problems. Numerical 
results of the considered algorithms show that comparing with the $\varepsilon$-subgradient algorithm, the nonsmooth trust region algorithm has a better performance in terms of the number of function evaluations. Moreover, comparing with the subgradient algorithm, the nonsmooth trust region method gives us a better approximation of the minimum value of the function for some examples.

\section{Acknowledgement}

The authors wish to thank R. Yousefpour and C. Lageman for useful discussion and providing their implementations of subgradient methods.

\section{Funding}

The work was supported by the Swiss National Fund (SNF) under grant number 140635.

\section{REFERENCES}

Absil, P. A. \& Baker, C. G. (2007) Trust-region methods on Riemannian manifolds. Found. Comput. Math., 7, 303-330.

Absil, P. A., Mahony, R. \& Sepulchre, R. (2008) Optimization Algorithm on Matrix Manifolds. Princeton: Princeton University Press.

Adler, R. L., Dedieu, J. P., Margulies, J. Y., Martens, M. \& Shub, M. (2002) Newton's method on Riemannian manifolds and a geometric model for the human spine. IMA J. Numer. Anal., 22, 359-390.

Afsari, B., Tron, R. \& Vidal, R. (2013) On the convergence of gradient descent for finding the Riemannian center of mass. SIAM J. Control Optim., 51, 2230-2260.

Akbari, Z., Yousefpour, R. \& Reza Peyghami, M. (2015) A new nonsmooth trust region algorithm for locally Lipschitz unconstrained optimization problems. J. Optim. Theory Appl., 164, 733-754.

Ashikhmin, A. E. \& Calderbank, A. R. (2010) Grassmannian packings from operator Reed-Muller codes. IEEE Trans. Inf. Theory, 56, 5689-5714.

Azagra, D. \& Ferrera, J. (2007) Applications of proximal calculus to fixed point theory on Riemannian manifolds. Nonlinear. Anal., 67, 154-174.

Azagra, D., Ferrera, J. \& López-Mesas, F. (2005) Nonsmooth analysis and Hamilton-Jacobi equations on Riemannian manifolds. J. Funct. Anal., 220, 304-361.

Basser, P. J., Mattiello, J. \& Bihan, D. L. (1994) MR diffusion tensor spectroscopy and imaging. Biophys. J., 66, 259-267.

Bento, G. C., Ferreira, O. P. \& Oliveira, P. R. (2010) Local convergence of the proximal point method for a special class of nonconvex functions on Hadamard manifolds. Nonlinear Anal., 73, 564-572.

Bento, G. C., Ferreira, O. P. \& Oliveira, P. R. (2012) Unconstrained steepest descent method for multicriteria optimization on Riemannian manifolds. J. Optim. Theory Appl., 154, 88-107.

Bento, G. C., Ferreira, O. P. \& Oliveira, P. R. (2015) Proximal point method for a special class of nonconvex functions on Hadamard manifolds. Optimi., 64, 289-319.

Bento, G. C. \& Melo, J. G. (2012) A subgradient method for convex feasibility on Riemannian manifolds. J. Optim. Theory Appl., 152, 773-785.

Borckmans, P. B., Easter Selvan, S., Boumal, N. \& Absil, P. A. (2014) A Riemannian subgradient algorithm for economic dispatch with valve-point effect. J. Comput. Appl. Math., 255, 848-866.

Canary, R. D., Epstein, D. B. A. \& Marden, A. (2006) Fundamentals of Hyperbolic Geometry: Selected Expositions. Cambridge: Cambridge University Press.

Conn, A. R., Gould, N. I. M. \& Toint, Ph. L. (2000) Trust Region Methods, SIAM Series on Optimization. Philadelphia: SIAM.

da Cruz Neto, J. X., Ferreira, O. P. \& Lucambio Perez, L. R. (1999) A proximal regularization of the steepest descent method in Riemannian manifold. Balkan J. Geom. Appl., 2, 1-8. 
da Cruz Neto, J. X., Lima, L. L. \& Oliveira, P. R. (1998) Geodesic algorithm in Riemannian manifolds. Balkan J. Geom. Appl., 2, 89-100.

Dennis, J. E., LI, S. B. \& TAPIA, R. A. (1995) A unified approach to global convergence of trust region methods for nonsmooth optimization. Math. Program., 68, 319-346.

Dirr, G., Helmke, U. \& Lageman, C. (2007) Nonsmooth Riemannian optimization with applications to sphere packing and grasping. Lagrangian and Hamiltonian Methods for Nonlinear Control 2006: Proceedings from the 3rd IFAC Workshop, Nagoya, Japan, 2006. Lecture Notes in Control and Information Sciences, vol. 366. Berlin: Springer.

Ferreira, O. P. \& Oliveira, P. R. (1998) Subgradient algorithm on Riemannian manifolds. J. Optim. Theory Appl., 97, 93-104.

Ferreira, O. P. \& Oliveira, P. R. (2002) Proximal point algorithm on Riemannian manifolds. Optimization, 51, 257-270.

Grohs, P., Hardering, H. \& SAnder, O. (2014) Optimal a priori discretization error bounds for geodesic finite elements. Found. Comput. Math., DOI: 10.1007/s10208-014-9230-z (last accessed 26 August 2015).

Grohs, P. \& Hosseini, S. (2015) $\varepsilon$-Subgradient algorithms for locally Lipschitz functions on Riemannian manifolds. Adv. Comput. Math., DOI: 10.1007/s10444-015-9426-z (last accessed 26 August 2015).

Helmke, U. \& Moore, J. (1994) Optimization and Dynamical System. London: Springer.

Hosseini, S. \& Pouryayevali, M. R. (2011) Generalized gradients and characterization of epi-Lipschitz sets in Riemannian manifolds. Nonlinear Anal., 74, 3884-3895.

Hosseini, S. \& Pouryayevali, M. R. (2013a) Euler characterization of epi-Lipschitz subsets of Riemannian manifolds. J. Convex. Anal., 20, 67-91.

Hosseini, S. \& Pouryayevali, M. R. (2013b) On the metric projection onto prox-regular subsets of Riemannian manifolds. Proc. Amer. Math. Soc., 141, 233-244.

KLingenberG, W. (1995) Riemannian Geometry. Walter de Gruyter Studies in Mathematics, vol. 1. Berlin, New York: Walter de Gruyter.

Kressner, D., Steinlechner, M. \& Vandereycken, B. (2014) Low-rank tensor completion by Riemannian optimization. BIT Numer. Math., 54, 447-468.

Lageman, C. \& Helmke, U. (2007) Optimization Methods for the Sphere Packing Problem on Grassmannians. Taming Heterogeneity and Complexity of Embedded Control, 393-408, ISTE, London.

Lang, S. (1999) Fundamentals of Differential Geometry. Graduate Texts in Mathematics, vol. 191. New York: Springer.

LEE, P. Y. (2005) Geometric optimization for computer vision. Ph.D. Thesis, Australian National University.

Li, C., Mordukhovich, B. S., Wang, J. \& Yao, J. C. (2011) Weak sharp minima on Riemannian manifolds. SIAM J. Optim., 21, 1523-1560.

Mahdavi-Amiri, N. \& Yousefpour, R. (2012) An effective nonsmooth optimization algorithm for locally Lipschitz functions. J. Optim. Theory Appl., 155, 180-195.

Mahony, R. E. (1996) The constrained Newton method on a Lie group and the symmetric eigenvalue problem. Linear Algebra Appl., 248, 67-89.

Ozbek, B. \& Ruyet, D. L. (2013) Feedback Strategies for Wireless Communication. Berlin, Heidelberg, New York: Springer

PaPa Quiroz, E. A., Quispe, E. M. \& Oliveira, P. R. (2008) Steepest descent method with a generalized Armijo search for quasiconvex functions on Riemannian manifolds. J. Math. Anal. Appl., 341, 467-477.

Pennec, X., Fillard, P. \& Ayache, N. (2006) A Riemannian framework for tensor computing. Int. J. Comput. Vis., 66, 41-66.

QI, C., Gallivan, K. A. \& Absil, P. A. (2010) Riemannian BFGS Algorithm with Applications. Recent Advances in Optimization and its Applications in Engineering, pp. 183-192. Available at http://link.springer.com/chapter/10.1007\%2F978-3-642-12598-0_16 (last accessed 26 August 2015).

QI, L. \& Sun, J. (1994) A trust region algorithm for minimization of locally Lipschitzian functions. Math. Program., 66, 25-43.

RidDELL, R. C. (1984) Minimax problems on Grassmann manifolds. Sums of eigenvalues. Adv. Math., 54, 107-199. 
RING, W. \& WiRTH, B. (2012) Optimization methods on Riemannian manifolds and their application to shape space. SIAM J. Optim., 22, 596-627.

Rudin, L., Osher, S. \& FAtemi, E. (1992) Nonlinear total variation based noise removal algorithms. Phys. D., 60, 259-268.

SANDER, O. (2010) Geodesic finite elements for Cosserat rods. Int. J. Numer. Methods Eng., 82, 1645-1670.

SARKIS, M. \& DiEPOLD, K. (2012) Camera-pose estimation via projective Newton optimization on the manifold. IEEE Trans. Image Processing., 21, 1729-1741.

Sмiтн, S. T. (1994) Optimization techniques on Riemannian manifolds. Fields Inst. Commun., 3, 113-146.

Udriste, C. (1994) Convex Functions and Optimization Methods on Riemannian Manifolds. Dordrecht, The Netherlands: Kluwer Academic Publishers.

Usevich, K. \& Markovsky, I. (2014) Optimization on a Grassmann manifold with application to system identification. Automatica., 50, 1656-1662.

VANDEREYCKen, B. (2013) Low-rank matrix completion by Riemannian optimization. SIAM J. Optim., 23, $1214-1236$.

VANDEREyCKen, B. \& VANDEWALle, S. (2010) A Riemannian optimization approach for computing low-rank solutions of Lyapunov equations. SIAM. J. Matrix Anal. Appl., 31, 2553-2579.

Weinmann, A., Demaret, L. \& Storath, M. (2014) Total variation regularization for manifold-valued data. SIAM J. Imaging Sci., 7, 2226-2257.

Zhang, L. H. (2010) Riemannian Newton method for the multivariate eigenvalue problem. SIAM J. Matrix Anal. Appl., 31, 2972-2996. 